\title{
PREFACTIBILIDAD DE OBTENCIÓN DE ISOBUTANO Y PROPANO MEDIANTE GASES DE EXTRACCIÓN PETROLÍFERA EN COLOMBIA
}

PREFEASIBILITY FOR OBTAINING ISOBUTANE AND PROPANE VIA PETROLEUM EXTRACTION GASES IN COLOMBIA

\author{
Claudia Milena Moreno Carvajal* \\ Carlos Urrego Rodríguez**
}

FUNDACIÓN UNIVERSIDAD DE AMÉRICA

Recibido: 2 de septiembre de 2018 Aceptado: 24 de junio de 2019

DOI: https://doi.org/10.29097/23461098.310

\section{Resumen}

El isobutano y propano, obtenidos con gas natural y gas extraído del petróleo (GLP), pueden reemplazar refrigerantes sintéticos para reducir las emisiones de gases que agotan la capa de ozono y generan calentamiento global; por tal motivo se realizó una simulación en Aspen HYSYS para corroborar que es posible conseguir isobutano y propano al 99,5 \% en pureza. Las características del gas natural y del GLP se analizaron con cromatografías de gases proporcionadas por las empresas Gas Natural Fenosa y Almagas, tomando el menor promedio de los datos de isobutano y propano para determinar la pureza de cada uno de los compuestos por obtener. Por medio del análisis PUGH se seleccionaron cuatro métodos: directo, indirecto, membranas inorgánicas porosas y lecho fijo de adsorción para analizar la separación de isobutano y propano al 99,5 \% en pureza con gas natural y GLP. Finalmente se realizó la ingeniería conceptual de la separación con la simulación en Aspen HYSYS V 9.0, obteniendo en pureza 99,5 \% de propano; 49,62 \% de isobutano, y 49,38 \% de n-butano al utilizar gas natural, el cual se componía de metano $\left(\mathrm{CH}_{4}\right)$ en un volumen de $84 \%$; etano $\left(\mathrm{C}_{2} \mathrm{H}_{6}\right)$ del 3 al $8 \%$; propano $\left(\mathrm{C}_{3} \mathrm{H}_{6}\right)$ 1-2 \%, y butano $\left(\mathrm{C}_{4} \mathrm{H}_{10}\right)$ menor de $1 \%$. También se observaron cantidades muy pequeñas de compuestos más pesados como hexano $\left(\mathrm{C}_{6} \mathrm{H}_{14}\right)$, heptano $\left(\mathrm{C}_{7} \mathrm{H}_{16}\right)$ y octanos $\left(\mathrm{C}_{8} \mathrm{H}_{18}\right)$ con un porcentaje en volumen menor de $1 \%$. Adicionalmente se encontraron impurezas como dióxido de carbono $\left(\mathrm{CO}_{2}\right)$ con $1-2 \%$; sulfuro de hidrógeno $\left(\mathrm{H}_{2} \mathrm{~S}\right)$ menor de $1 \%$ y nitrógeno $\left(\mathrm{N}_{2}\right)$ con $1 \%$; propano 99,9 \%; isobutano 1,98 \%, y 97,45 \% de n-butano. El GLP se componía de nitrógeno $\left(\mathrm{N}_{2}\right)$ al 0,05 \%; etano $\left(\mathrm{C}_{2} \mathrm{H}_{6}\right)$ al 2,07 \%; propano $\left(\mathrm{C}_{3} \mathrm{H}_{8}\right)$ al 66,65 \%; isobutano $\left(\mathrm{i}-\mathrm{C}_{4} \mathrm{H}_{10}\right)$ al 16,46 \%; n-butano $\left(\mathrm{n}-\mathrm{C}_{4} \mathrm{H}_{10}\right)$ al $14,87 \%$, e isopentano $\left(\mathrm{i}-\mathrm{C}_{5} \mathrm{H}_{12}\right)$.

Palabras clave: isobutano, prefactibilidad, propano, refrigerantes.

\footnotetext{
* Estudiante de Ingeniería Química, Grupo de Investigación en Térmicas y Fluidos, GITEF.

凶. claudia.moreno@estudiantes.uamerica.edu.co

** Ingeniero mecánico. Coinvestigador Grupo de Investigación en Térmicas y Fluidos, GITEF.
} 


\section{Abstract}

Isobutane and propane obtained from natural gas and liquefied petroleum gas (LPG) can be used to replace synthetic refrigerants, reducing emissions of ozone-depleting gases that generate global warming. For this reason, a simulation was carried out using Aspen HYSYS to corroborate that it is possible to obtain isobutane and propane at 99.5\% purity. Properties of natural gas and LPG were analyzed with gas chromatography provided by Gas Natural Fenosa and Almagas, taking the lowest average of isobutane and propane data to determine the purity of each of the compounds to be obtained. Using PUGH analysis, four methods were selected: direct, indirect, inorganic porous membranes, and fixed adsorption bed to analyze the separation of isobutane and propane at 99,5\% purity with natural gas and LPG. Finally, the conceptual engineering of the separation was carried out with the simulation in Aspen HYSYS V 9.0, obtaining $99.5 \%$ propane, $49.62 \%$ isobutane, and $49.38 \%$ n-butane in purity, when using natural gas, which is composed of methane $(\mathrm{CH} 4)$ in a volume of $84 \%$, ethane (C2H6) at 3-8\%, propane (C3H6) 1-2\%, and butane (C4H10) less than 1\%. Small amounts of heavier compounds such as hexane (C6H14), heptane (C7H16), and octane $(\mathrm{C} 8 \mathrm{H} 18)$ were also observed with a volume percentage lower than $1 \%$. Additionally, some impurities were found in this gas such as carbon dioxide (CO2) with 1-2\%, hydrogen sulfide (H2S) with less than 1\%, and nitrogen (N2) with 1\%, 99.9\% propane, $1.98 \%$ isobutane, and $97.45 \%$ n-butane. LPG is composed of nitrogen (N2) at 0,05\%, ethane (C2H6) at 2.07\%, propane (C3H8) at $66.65 \%$, isobutane (i-C4H10) at $16.46 \%$, n-butane (n-C4H10) at 14.87\%, and isopentane (i-C5H12).

Keywords: isobutane, pre-feasibility, propane, refrigerants.

\section{INTRODUCCIÓN}

Dada la necesidad de eliminar las emisiones de gases que dañan la capa de ozono y generan calentamiento global, causado en parte por los refrigerantes sintéticos utilizados en las últimas décadas, se hace importante la determinación de la prefactibilidad de la obtención de isobutano y propano al 99,5 \% en pureza para ser usados como refrigerantes naturales. Para tal fin se tomaron datos de cromatografías, proporcionadas por las empresas extractoras tanto de gas natural como de GLP, y se simularon con ayuda del Aspen HYSYS (Hamid, M. K., 2007), una herramienta para simulación de los equipos requeridos para este proyecto, que determina la viabilidad de obtener estos dos refrigerantes en Colombia.

Con la entrada en vigor del Protocolo de Montreal en 1987 (Programa de las Naciones Unidas para el Medio Ambiente, 2009), las sustancias refrigerantes clorofluorocarbonos - CFC - reductoras de la capa de ozono fueron retiradas del mercado. En la etapa dos de este protocolo, los refrigerantes hidroclorofluorocarbonos - HCFC - entraron en proceso de eliminación; la COP 21 (Conference of the Parties [Conferencia de las Naciones Unidas sobre Cambio Climático]) de 2015, la COP 22 de 2016 (Organización de las Naciones Unidas [ONU], 2015) y la Enmienda de Kigali de 2016 (Ley 1970 de 2019) generaron un plan de eliminación de las sustancias hidrofluorocarbonadas - HFC - causantes de calentamiento global. Estos hechos 
originan el proyecto "Prefactibilidad de obtención de isobutano y propano mediante gases de extracción petrolífera en Colombia”, buscando la oportunidad de producir en el país refrigerantes naturales hidrocarbonados - HC—, los cuales poseen mínima huella de calentamiento global y cero agotamientos de la capa de ozono (según la Unidad Técnica Ozono UTO como oficina implementadora del Protocolo de Montreal).

El alcance del proyecto se determinó en el análisis de las cromatografías del gas natural y GLP de extracción petrolífera nacional, en la selección del método de separación más viable para la obtención de estas sustancias, la simulación de la separación de los compuestos, y el desarrollo de ingeniería conceptual de la separación para la obtención de isobutano y propano.

\section{METODOLOGÍA}

Para esta investigación se usaron cromatografías, suministradas por Gas Natural Fenosa y Almagas, que muestran las cantidades de cada uno de los compuestos que conforman la materia prima de gas natural y GLP. De estas cromatografías se seleccionaron las de menor promedio de isobutano y propano. Se hizo así con el fin de mostrar que se pueden obtener, con cantidades bajas, los porcentajes de pureza planteados, como se observa en las Tablas 1 y 2. Estos datos se programaron en el simulador Aspen HYSYS para determinar el porcentaje en pureza y las mínimas cantidades por obtener de dichas sustancias.

\section{Tabla 1}

Calidad del gas natural tomado del 10 al 16 de abril de 2017

\begin{tabular}{lc}
\hline Gas Usme & Cantidad másica promedio \\
\hline Nitrógeno $\left(\mathrm{N}_{2}\right)$ & 0,8129 \\
\hline Dióxido de carbono $\left(\mathrm{CO}_{2}\right)$ & 3,5957 \\
\hline Metano $\left(\mathrm{CH}_{4}\right)$ & 82,8037 \\
\hline Etano $\left(\mathrm{C}_{2} \mathrm{H}_{6}\right)$ & 10,0642 \\
\hline Propano $\left(\mathrm{C}_{3} \mathrm{H}_{8}\right)$ & 2,0449 \\
\hline I-butano $\left(\mathrm{i}-\mathrm{C}_{4} \mathrm{H}_{10}\right)$ & 0,2511 \\
\hline N-butano $\left(\mathrm{n}-\mathrm{C}_{4} \mathrm{H}_{10}\right)$ & 0,2435 \\
\hline I-pentano $\left(\mathrm{i}-\mathrm{C}_{5} \mathrm{H}_{12}\right)$ & 0,0441 \\
\hline N-pentano $\left(\mathrm{n}-\mathrm{C}_{5} \mathrm{H}_{12}\right)$ & 0,0241 \\
\hline N-hexano $\left(\mathrm{n}-\mathrm{C}_{6}+\right)$ & 0,1158 \\
\hline
\end{tabular}


Moreno Carvajal y Urrego Rodríguez

\begin{tabular}{ll}
\hline Neopentano (neo- $\left.\mathrm{C}_{5} \mathrm{H}_{12}\right)$ & 0,0000 \\
\hline BTUs @ 14.65 Dry (poder calorífico) & 1091,23 \\
\hline Densidad relativa de la composición & 0,6709 \\
\hline Densidad real $\left(\mathrm{kg} / \mathrm{m}^{3}\right)$ & 0,8260 \\
\hline
\end{tabular}

Nota. Tabla elaborada con cromatografías proporcionadas por Gas Natural Fenosa.

Tabla 2

Calidad del GLP tomado el 11 de julio de 2017

\begin{tabular}{ll}
\hline Componente & Cantidad másica promedio \\
\hline Nitrógeno & 0,0000 \\
\hline Metano & 0,0000 \\
\hline Dióxido de carbono & 0,0000 \\
\hline Etano & 2,7217 \\
\hline Propano & 17,8305 \\
\hline I-butano & 15,4109 \\
\hline N-butano & 62,8415 \\
\hline I-pentano & 1,1325 \\
\hline N-pentano & 0,0000 \\
\hline Hexano & 0,0629 \\
\hline Heptanos & 0,0000 \\
\hline
\end{tabular}

Nota. Tabla elaborada con cromatografías proporcionadas por Almagas.

Entre la bibliografía especializada se encuentra el artículo “Applied digital library project management: Using Pugh matrix analysis in complex decision-making situations" (Cervone, H. F., 2009), donde se identificaron cuatro procedimientos de separación de gases: directo, indirecto, membranas inorgánicas porosas y lecho fijo de absorción; estos métodos se referenciaron por los ingenieros Rainer Maldonado, Andrea Angulo, Katherine Cival, Ricardo García y Marianne Suárez, y se aplicaron para la materia prima GLP en su proyecto "Purificación de propano como refrigerante R290" (Maldonado, R., 2013). La selección del método de separación para la obtención de isobutano y propano al 99,5 \% en pureza del gas natural y GLP se realizó con un análisis de la matriz PUGH (Cervone, H. F., 2009), una herramienta cuantitativa que permite comparar opciones mediante un arreglo multidimensional (matriz de decisiones). 
La selección del modo de separación más factible para obtener isobutano y propano al 99.5 \% usó el método PUGH, una herramienta cuantitativa que permite comparar opciones mediante un arreglo multidimensional (matriz de decisiones). Para aplicarla se procede así:

- Se toma la primera columna del cuadro llamado conceptos y se analiza criterio por criterio, en este caso son: energía utilizada en cada proceso, pureza requerida, cantidad del componente y utilización de otro método de separación.

- Para cada concepto, en este caso la destilación directa, destilación indirecta, membranas inorgánicas porosas y lecho fino de adsorción, la alternativa actual se evaluó con respecto a la línea base como: mejor (+1), el mismo (0) o peor (-1). Los números positivos reflejan calificaciones deseables, y los negativos, calificaciones indeseables.

- El concepto que obtenga un resultado mayor, producto del balance entre aspectos positivos y negativos, se considerará la mejor solución.

- Para completar la clasificación, se generaron tres puntajes: el mayor, el número de puntuaciones negativas y las puntuaciones totales generales. El total general se calcula restando el número de puntajes negativos del número de mayor puntaje.

En la Tabla 3 se muestran los datos recolectados para escoger el método de separación más viable, y en la tabla 4 se escogió la mejor opción para el proceso de separación y obtención de isobutano y propano con el requerimiento planteado de 99,5 \%. En la Tabla 4 se presenta el análisis de los diferentes métodos de separación:

- Destilación directa. Se separa el componente más volátil en la primera columna y es improbable la recuperación del propano e isobutano (ver Figura 1).

- Destilación por método indirecto. Es la separación del componente más pesado; en este caso sería el isobutano $\left(\mathrm{iC}_{4}\right)$ y el n-butano $\left(\mathrm{nC}_{4}\right)$ (ver Figura 2).

- Membranas inorgánicas porosas. Separación de los componentes sin adición de químicos y con un bajo de consumo de energía. Funciona como un filtro donde ciertas sustancias atraviesan la membrana, y otras quedan atrapadas en ella, se utilizan para operaciones unitarias de separación que pueden ser poliméricas o inorgánicas (ver Figura 3).

- Lecho fijo de absorción. Opera con una columna adicional donde se regenera el sólido adsorbente, por remoción de absorbato con una corriente a baja presión; el permeado obtenido de la pervaporación puede inyectarse como corriente para regenerar el lecho saturado (ver Figura 4). 
Figura 1

Proceso de destilación de forma directa

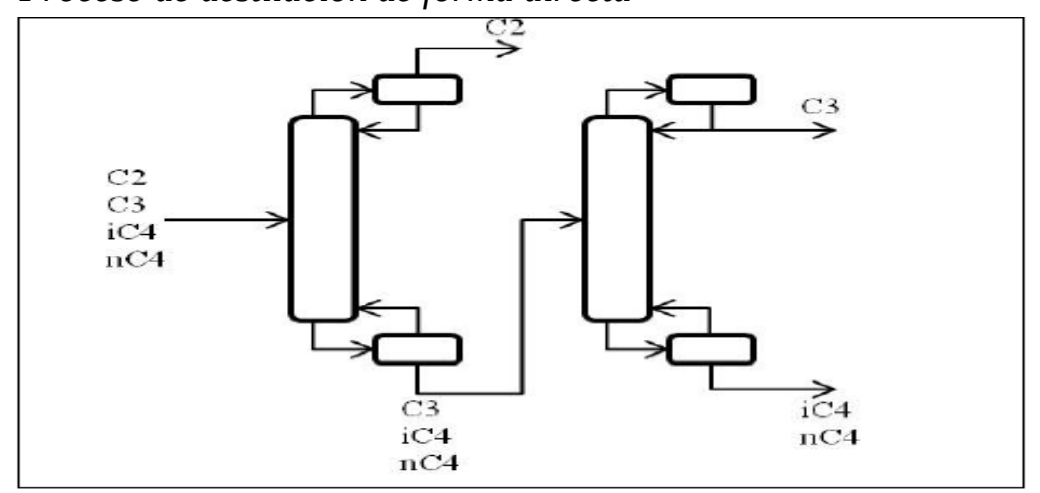

Nota. Esquema tomado de Maldonado, R., et al. (2017).

Figura 2

Proceso de destilación forma indirecta, integración térmica

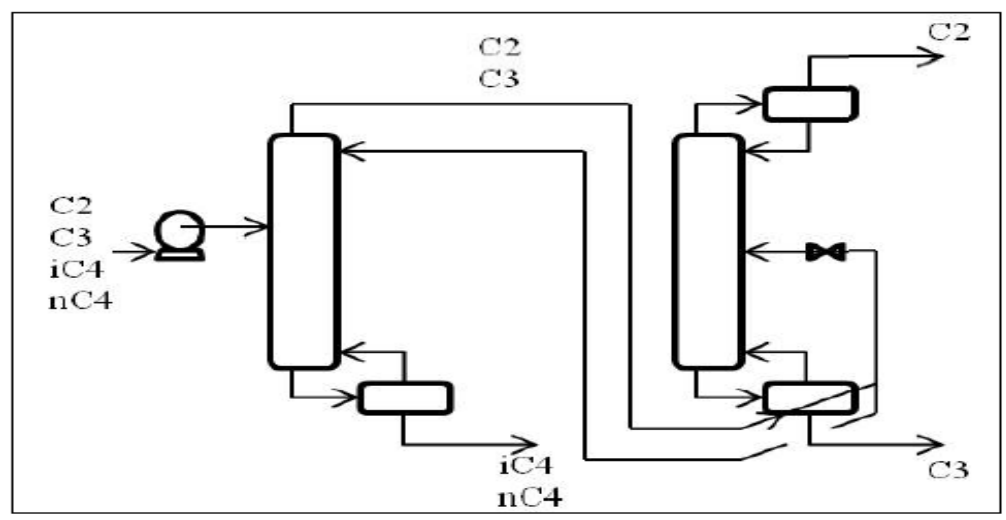

Nota. Esquema tomado de Maldonado, R., et al. (2013).

Figura 3

Obtención de isobutano y propano por membranas

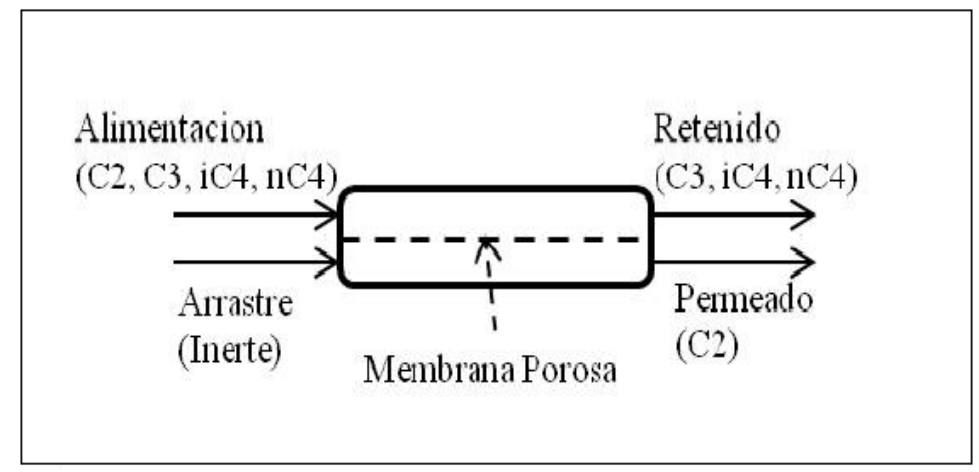

Nota. Esquema tomado de Maldonado, R., et al. (2013). 


\section{Figura 4}

Procesos de pervaporación y adsorción de presión alternante de lecho fijo para purificación del propano

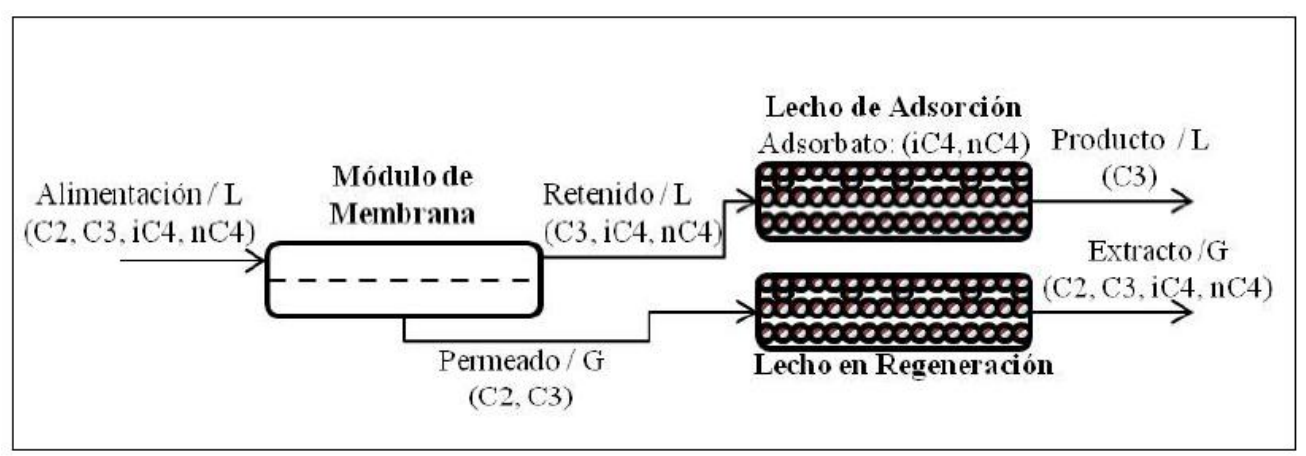

Nota. Esquema tomado de Maldonado, R., et al. (2013).

Se observa que el método más favorable para la obtención de isobutano y propano con gas natural y GLP es la destilación indirecta; así se concluyó, porque este proceso arrojó el mayor puntaje positivo que, además, cumple con el propósito de alcanzar el 99,5 \% en pureza de las sustancias.

\section{Tabla 3}

Datos de los procesos de separación

\begin{tabular}{lllll} 
& Conceptos & & & \\
\hline Criterios & Destilación & Destilación & Membranas & Lecho fino de \\
& directa & indirecta & inorgánicas porosas & adsorción \\
\hline Energía utilizada & N/A & $236 \mathrm{~kW}$ & No utiliza & No utiliza \\
\hline Porcentaje pureza & $85 \%$ & $99,5 \%$ & $85 \%$ & $95 \%$ \\
deseada & & & & No utiliza \\
\hline Cantidad del & $80 \mathrm{kmol} / \mathrm{h}$ & $90 \mathrm{kmol} / \mathrm{h}$ & $80 \mathrm{kmol} / \mathrm{h}$ & \\
componente & & & & Sí \\
\hline Utilización de otro & & & Sí & \\
método de separación & No & No & & \\
\hline
\end{tabular}

Nota. Tabla de elaboración propia basada en Maldonado, R., et al. (2013). 
Moreno Carvajal y Urrego Rodríguez

Tabla 4

Método PUHG para la selección del modo de separación para isobutano y propano

\begin{tabular}{|c|c|c|c|c|c|c|c|}
\hline & $\begin{array}{c}\text { Energía } \\
\text { utilizada } \\
(\mathbf{k W})\end{array}$ & $\begin{array}{c}\text { Pureza } \\
(\%)\end{array}$ & $\begin{array}{l}\text { Cantidad del } \\
\text { componente }\end{array}$ & $\begin{array}{c}\text { Utilización } \\
\text { del método de } \\
\text { separación }\end{array}$ & Total + & Total - & $\begin{array}{c}\text { Total } \\
\text { general }\end{array}$ \\
\hline \multicolumn{8}{|l|}{ Factor 1} \\
\hline $\begin{array}{l}\text { Destilación } \\
\text { directa }\end{array}$ & -1 & -1 & 0 & 0 & & -2 & -2 \\
\hline \multicolumn{8}{|l|}{ Factor 2} \\
\hline $\begin{array}{l}\text { Destilación } \\
\text { indirecta }\end{array}$ & +1 & +1 & 0 & 0 & 2 & & 2 \\
\hline \multicolumn{8}{|l|}{ Factor 3} \\
\hline $\begin{array}{l}\text { Membranas } \\
\text { inorgánicas }\end{array}$ & 0 & -1 & -1 & -1 & & -3 & -3 \\
\hline \multicolumn{8}{|l|}{ Factor 4} \\
\hline $\begin{array}{l}\text { Lecho fijo } \\
\text { de adsorción }\end{array}$ & 0 & -1 & -1 & -1 & & -3 & -3 \\
\hline
\end{tabular}

Nota. Tabla de elaboración propia basada en Cervone, H. F. (2009).

Escogido el método de separación, se opera el Aspen HYSYS V 9.0, una herramienta utilizada para simulación de los diferentes equipos requeridos para el desarrollo de esta investigación, donde se siguió el proceso de separación de gas natural (ver figura 5), el cual, por contener varios compuestos, precisó de distintos equipos, entre los que se encuentran: el compresor que aumenta la presión para que la materia prima llegue a su saturación; tres enfriadores por los que pasa la materia prima con el objetivo de que alcance el estado líquido para efectuar la separación; una primera columna llamada flash, donde las diferentes volatilidades de los compuestos empiezan a efectuar la separación. Al no evidenciarse mayor separación en esta columna, se llevó la corriente de la materia prima a una segunda columna llamada desmetanizadora, donde se apreció separación de los compuestos y perdida de metano. También se necesitó una bomba para que la velocidad de flujo de la materia prima aumentara; posteriormente se pasó a una tercera columna llamada desetanizadora, en la que se siguieron separando los compuestos de la materia prima y se eliminó el etano; una válvula de expansión con el fin de bajar la presión; una cuarta columna llamada despropanizadora, en donde se obtuvo propano y se disminuyeron trazas de las otras materias primas; una segunda válvula de expansión para seguir bajando la presión y la temperatura, y ,finalmente, una quinta columna llamada desbutanizadora, en donde no solo se logró obtener isobutano sino también n-butano. Posteriormente, se simuló el GLP (ver figura 6), ingresando a las propiedades del programa, en la carpeta paquete de fluidos, los componentes del GLP en estado gaseoso: dióxido de carbono, nitrógeno, etano, metano, propano, ibutano, n-butano, i-pentano, n-pentano y n-hexano, y se añadieron los datos de la tabla 
2 con un paquete de propiedades de Peng-Robinson, un modelo ideal para el cálculo de la densidad de los líquidos en sistemas de hidrocarburos (Wikispaces, 2017).

\section{Figura 5}

Diagrama de flujo del proceso de separación del gas natural

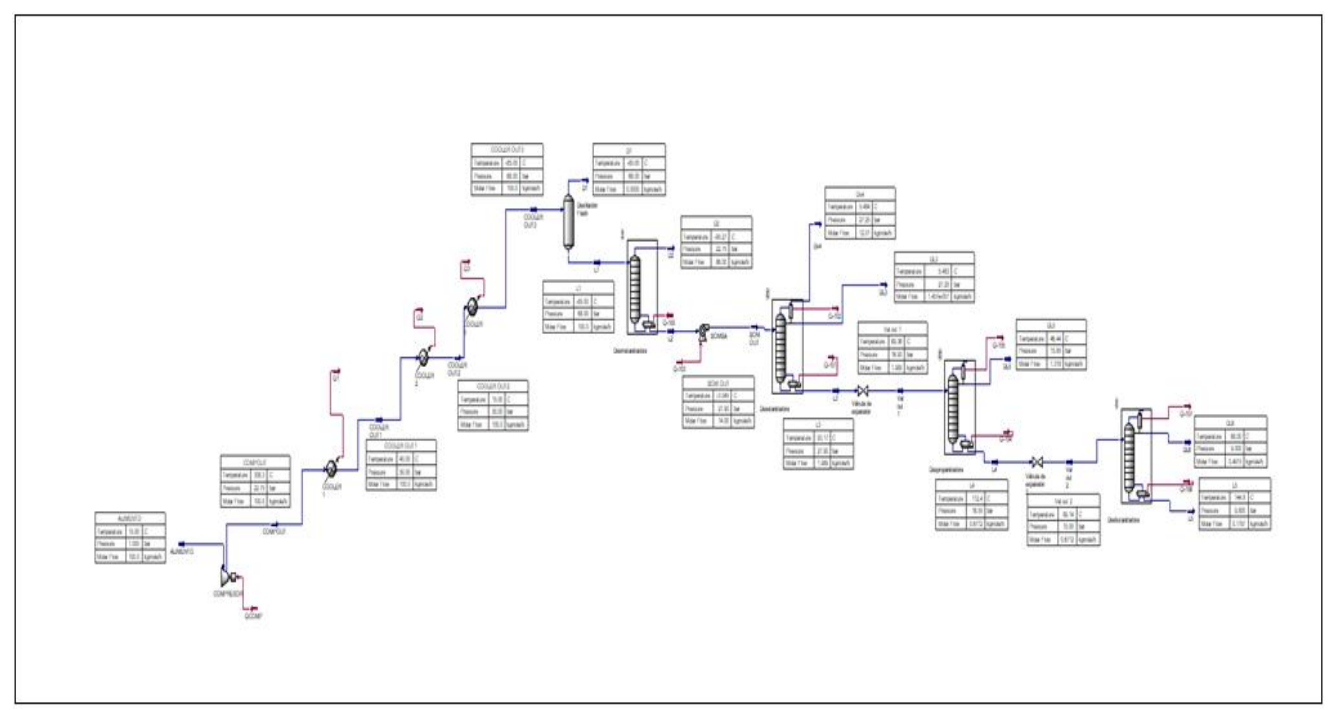

Nota. Esquema de elaboración propia mediante Aspen Hysys V 9.0.

Figura 6

Diagrama del proceso de separación del GLP

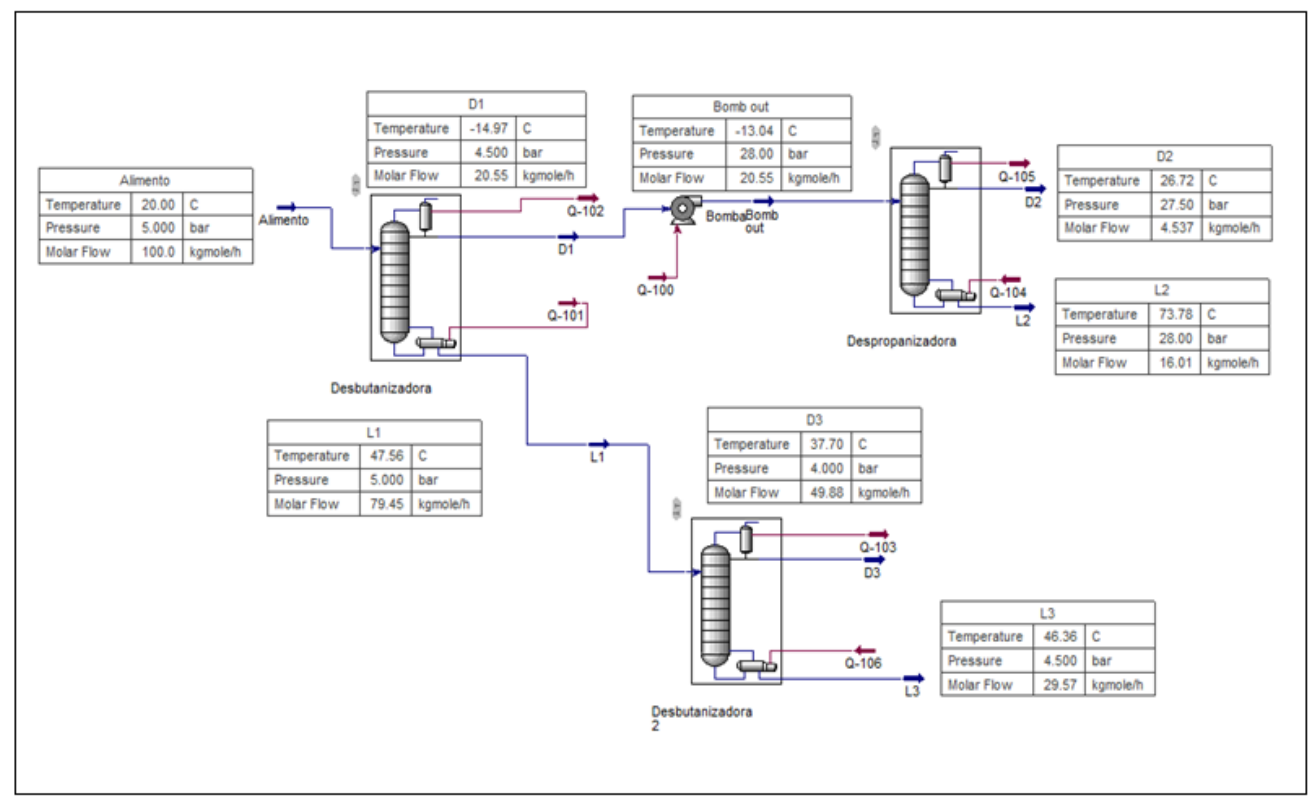

Nota. Esquema de elaboración propia mediante Aspen Hysys V 9.0. 
Se creó una corriente de materia nombrada alimento, adonde se ingresaron las especificaciones de flujo $100 \mathrm{kmol} / \mathrm{h}$, temperatura $20^{\circ} \mathrm{C}$, presión 5 bares y las composiciones tomadas de las cromatografías de la tabla 2 de la materia prima del GLP; en la primera columna se extrajeron los compuestos de mayor flujo, en este caso los butanos, por eso el uso de una desbutanizadora, con la que, por las cimas o parte superior de la columna, se extraen etano y propano, para luego ser separados.

La corriente que fue separada del propano y etano se envió a una bomba para que aumentara en la velocidad de flujo, $y$, seguidamente, a una segunda columna llamada despropanizadora para separar el propano del etano; en la tercera columna, llamada desbutanizadora, se separaron los butanos del i-pentano y n-hexano, y se obtuvieron isobutano y n-butano.

\section{RESULTADOS Y DISCUSIÓN}

Habiendo tomado el menor promedio de los compuestos de las cromatografías de gas natural y GLP, y escogido el método de separación indirecto para la obtención de isobutano y propano al 99,5 \% en pureza, se continuó con el ambiente de simulación, donde se evidenció que en el gas natural comienza a efectuarse la separación de los compuestos de la materia prima en la columna desmetanizadora, mas no se encuentran trazas de metano ni de nitrógeno, como se observa en la Tabla 5:

\section{Tabla 5}

Composiciones en la corriente de fondos o L2

\begin{tabular}{lcc}
\hline Compuestos & Fracciones molares & Fase de vapor \\
\hline Nitrógeno & 0,0000 & 0,0000 \\
\hline Dióxido de carbono & 0,1641 & 0,1641 \\
\hline Metano & 0,0000 & 0,0000 \\
\hline Etano & 0,6434 & 0,6434 \\
\hline Propano & 0,1441 & 0,1441 \\
\hline I-butano & 0,0179 & 0,0179 \\
\hline N-butano & 0,0174 & 0,0174 \\
\hline I-pentano & 0,0031 & 0,0031 \\
\hline N-pentano & 0,0017 & 0,0017 \\
\hline N-hexano & 0,0083 & 0.0000 \\
\hline
\end{tabular}

Nota. Tabla de elaboración propia mediante el simulador Aspen HYSYS V 9.0. 
Esta corriente L2 se envía a la columna desetanizadora para retirar el etano y el CO2, eliminando los compuestos indeseados (ver Tabla 6).

Para la eliminación de los compuestos restantes como i-pentano, n-pentano y n-hexano, se programaron las columnas despropanizadora y desbutanizadora; mientras que, para purificar y obtener el propano, se lograron 99,5 \% de propano; 49,62 \% de i-butano, y $49,38 \%$ de n-butano en pureza, como se observa en las Tablas 7 y 8.

Tabla 6

Composiciones de salida en la corriente de fondos L3

\begin{tabular}{lccc}
\hline Compuestos & Fracciones molares & Fase de vapor & Fase líquida \\
\hline Nitrógeno & 0,0000 & 0,0000 & 0,0000 \\
\hline CO2 & 0,0000 & 0,0000 & 0,0000 \\
\hline Metano & 0,0000 & 0,0000 & 0,0000 \\
\hline Etano & 0,0001 & 0,0003 & 0,0001 \\
\hline Propano & 0,6594 & 0,7869 & 0,6594 \\
\hline I-butano & 0,1258 & 0,0996 & 0,1258 \\
\hline N-butano & 0,1222 & 0,0839 & 0,1222 \\
\hline I-pentano & 0,0222 & 0,0100 & 0,0222 \\
\hline N-pentano & 0,0121 & 0,0049 & 0,0121 \\
\hline N-hexano & 0,0582 & 0,0143 & 0,0582 \\
\hline
\end{tabular}

Nota. Tabla de elaboración propia mediante el simulador Aspen HYSYS V 9.0.

Tabla 7

Composición en la salida de la despropanizadora en el destilado DL5

\begin{tabular}{lll}
\hline Compuestos & Fracciones molares & Fase líquida \\
\hline Etano & 0,0002 & 0,0002 \\
\hline Propano & 0,9948 & 0,9948 \\
\hline I-butano & 0,0048 & 0,0048 \\
\hline
\end{tabular}


Moreno Carvajal y Urrego Rodríguez

\begin{tabular}{lcc}
\hline N-butano & 0,0002 & 0,0002 \\
\hline I-pentano & 0,0000 & 0,0000 \\
\hline N-pentano & 0,0000 & 0,0000 \\
\hline N-hexano & 0,0000 & 0,0000 \\
\hline
\end{tabular}

Nota. Tabla de elaboración propia mediante el simulador Aspen HYSYS V 9.0.

Tabla 8

Composición a la salida de la destilación DL6 en la desbutanizadora

\begin{tabular}{lcc}
\hline Compuestos & Fracciones molares & Fase líquida \\
\hline Propano & 0,0011 & 0,0011 \\
\hline I-butano & 0,4962 & 0,4962 \\
\hline N-butano & 0,4938 & 0,4938 \\
\hline I-pentano & 0,0087 & 0,0087 \\
\hline N-pentano & 0,0002 & 0,0002 \\
\hline
\end{tabular}

Nota. Tabla de elaboración propia mediante el simulador Aspen HYSYS V 9.0.

En el proceso de separación del GLP, se comenzó con una columna desbutanizadora a fin de separar los butanos (i-butano y n-butano) en los fondos, como se aprecia en la Tabla 9; además, como el GLP no contiene sustancias como CO2, N2, metano, ipentano, n-pentano ni n-hexano, la obtención del propano es más fácil y no requiere de muchos equipos para la efectiva separación. Así se observa en la Tabla 10.

Tabla 9

Composiciones de la salida en el fondo L1 en la desbutanizadora

\begin{tabular}{lcc}
\hline Compuestos & Fracciones molares & Fase líquida \\
\hline Propano & 0,109404 & 0,109404 \\
\hline I-butano & 9,039443 & 9,039443 \\
\hline N-butano & 36,911336 & 36,911336 \\
\hline I-pentano & 0,072156 & 0,072156 \\
\hline N-pentano & 0,0000 & 0,0000 \\
\hline
\end{tabular}




\begin{tabular}{lll}
\hline N-hexano & 0,051707 & 0,051707
\end{tabular}

Nota. Tabla de elaboración propia mediante el simulador Aspen HYSYS V 9.0.

\section{Tabla 10}

Composiciones de la salida en el fondo L1 en la desbutanizadora

\begin{tabular}{lcc}
\hline Compuestos & Fracciones molares & Fase líquida \\
\hline Etano & 0,1339 & 0,1339 \\
\hline Propano & 0,8651 & 0,8651 \\
\hline I-butano & 0,0010 & 0,0010 \\
\hline N-butano & 0,0001 & 0,0001 \\
\hline I-pentano & 0,0000 & 0,0000 \\
\hline N-pentano & 0,0000 & 0,0000 \\
\hline N-hexano & 0,0000 & 0,0000 \\
\hline
\end{tabular}

Nota. Tabla de elaboración propia mediante el simulador Aspen HYSYS V 9.0.

Después de la desbutanizadora se utilizó la despropanizadora, en cuyos fondos se obtuvo propano al 99,9 \% en pureza, como se observa en la Tabla 11:

\section{Tabla 11}

Condiciones de salida en los fondos L2 de la despropanizadora

\begin{tabular}{lcc}
\hline Compuestos & Fracciones molares & Fase líquida \\
\hline Etano & 0,0002 & 0,0002 \\
\hline Propano & 0,9985 & 0,9985 \\
\hline I-butano & 0,0012 & 0,0012 \\
\hline N-butano & 0,0001 & 0,0001 \\
\hline I-pentano & 0,0000 & 0,0000 \\
\hline
\end{tabular}

Nota. Tabla de elaboración propia mediante el simulador Aspen HYSYS V 9.0. 
Finalmente, de la corriente L1 resultante de la primera columna desbutanizadora se pasó a una segunda columna desbutanizadora para separar los butanos, y se obtuvo $97,47 \%$ de n-butano y 1,98 \% de i-butano en pureza, como se observa en la tabla 12 :

\section{Tabla 12}

Composiciones en la corriente de salida en desbutanizadora en fondos D3

\begin{tabular}{lll}
\hline Compuestos & Fracciones molares & Fase líquida \\
\hline Propano & 0,0000 & 0,0000 \\
\hline I-butano & 0,0198 & 0,0198 \\
\hline N-butano & 0,9747 & 0,9747 \\
\hline I-pentano & 0,0034 & 0,0034 \\
\hline N-pentano & 0,0000 & 0,0000 \\
\hline N-hexano & 0,0020 & 0,0020 \\
\hline
\end{tabular}

Nota. Tabla de elaboración propia mediante el simulador Aspen HYSYS V 9.0.

\section{CONCLUSIONES}

Se analizaron cuatro posibles separaciones con el método PUGH (ver tablas 3 y 4); se seleccionó la destilación indirecta, mostrado más viabilidad por el ahorro de energía sin la necesidad de otro método adicional para la obtención de isobutano y propano.

Se tomaron los datos de menor cantidad promedio de isobutano y propano (ver tabla 1 y 2) de las cromatografías suministradas por las empresas extractoras de estos gases, para luego ambientarse mediante simulación en Aspen Hysys V 9.0.

Se planteó una pureza mínima de 99,5 \% de los compuestos propano e isobutano, y se obtuvo 99,5 \% en pureza del primero y 49,62 \% del segundo, utilizando la materia prima gas natural por medio de la simulación elaborada. Asimismo, utilizando la materia prima GLP el porcentaje fue de 99,5 \% en pureza de propano; 49,62 \% de isobutano, y 49,38 \% de n-butano. Se logró demostrar que por cada $100 \mathrm{~kg} / \mathrm{h}$ de gas natural y GLP es viable la obtención $58,22 \mathrm{~kg} / \mathrm{h}$ de propano y $26,22 \mathrm{~kg} / \mathrm{h}$ de butano para el gas natural; para el GLP resultaron $16,01 \mathrm{~kg} / \mathrm{h}$ de propano y $29,57 \mathrm{~kg} / \mathrm{h}$ de butano.

\section{Referencias}

Cervone, H. F. (2009). Applied digital library project management: Using Pugh matrix analysis in complex decision-making situations. OCLC Systems \& Services: 
International Digital Library Perspectives, 25, pp. 284-294. DOI: 10.1108/10650750911001815.

Fronti de García, L., Fernández Cuesta, C. (2007). El Protocolo de Kioto y los costos ambientales. Revista del Instituto Internacional de Costos, No. 1, enerofebrero 2007, pp. 9-31.

http://www.revistaiic.org/articulos/num1/articulo1_esp.pdf

Hamid, M. K. (2007). HYSYS®: An Introduction to Chemical Engineering Simulation. Universiti Teknologi Malaysia.

http://eprints.utm.my/id/eprint/3030/2/HYSYS_for_UTM_Degree\%2B\%2B Program.pdf

Ley 1970 de 2019. Por medio de la cual se aprueba la "Enmienda de Kigali al Protocolo de Montreal”, adoptada el 15 de octubre de 2016 en Kigali, Ruanda. 12 de julio de 2019. D. O. No. 51.012.

Maldonado, R., Ángulo, A., Cival, K., García, R., Suárez, M. (2013). Procesos para purificación de propano como refrigerantes R290. Revista de la Facultad de Ingeniería U. C. V, 29(1), pp. 101-114.

http://ve.scielo.org/pdf/rfiucv/v29n1/art12.pdf

Organización de la Naciones Unidas, (ONU) (2015). Convención Marco sobre el Calentamiento Climático. ONU.

https://unfccc.int/resource/docs/2015/cop21/spa/109s.pdf

Secretaría del Convenio de Viena para la Protección de la Capa de Ozono, Protocolo de Montreal relativo a las sustancias que agotan la capa de ozono, Programa de las Naciones Unidad para el Medio Ambiente. (2009). Manual del Protocolo de Montreal relativo a las sustancias que agotan la capa de ozono. Programa de las Naciones Unidas para el Medio Ambiente.

https://www.car.gov.co/uploads/files/5b59e47ccaaae.pdf

Wikispaces (2017). Modelos de Hysys.

https://simulacionprocesos.wikispaces.com/Modelos+del+Hysys 